\title{
TREM-1 Contributes to Inflammation in IgA Nephropathy
}

\author{
Yan-Feng Zhao Li Zhu Li-Jun Liu Su-Fang Shi Ji-Cheng Lv Hong Zhang \\ Renal Division, Department of Medicine, Peking University First Hospital, Beijing, China; Peking University Institute \\ of Nephrology, Beijing, China; Key Laboratory of Renal Disease, Ministry of Health of China, Beijing, China; \\ Key Laboratory of Chronic Kidney Disease Prevention and Treatment (Peking University), Ministry of Education, \\ Beijing, China
}

\author{
Keywords \\ IgA nephropathy $\cdot$ TREM-1 - Circulating IgA1-containing \\ immune complexes
}

\begin{abstract}
Background: Circulating IgA1-containing immune complexes (clgA1) were shown to play important roles in IgA nephropathy (IgAN). They could induce the release of multiple inflammatory factors, including MCP-1 and IL-6, and elevated urinary inflammatory factors were also reported in patients with lgAN, which suggested that inflammation is a major contributor to kidney injury in IgAN. After the previous identification of the upregulated release of soluble triggering receptor expressed on myeloid cells-1 (sTREM-1) by mesangial cells under clgA1 challenge using cytokine array, in the present study, we further explored the role of TREM-1, an amplifier of inflammation, in clgA1-induced kidney injury. Methods: In total, 35 patients with IgAN and 17 healthy controls were enrolled. The clgA 1 was isolated from plasma and used to treat cultured mesangial cells. The mRNA expression of TREM- 1 as well as levels of sTREM-1, MCP-1, and IL- 6 in the mesangial cell supernatant and urine samples were detected. Results: We found that clgA1 from patients with IgAN
\end{abstract}

\section{KARGER}

(C) 2018 S. Karger AG, Basel

E-Mail karger@karger.com

www.karger.com/kdd could significantly upregulate the expression of TREM-1 in mesangial cells compared to healthy controls. The levels of $\triangle$ STREM-1 were positively correlated with MCP-1 levels in the mesangial supernatant. Similarly, higher urinary levels of sTREM-1 were also observed in patients with IgAN compared to healthy controls. Moreover, IgAN patients with detectable urinary STREM-1 presented with severe clinical and pathological manifestations, including higher IgA and lower eGFR levels, compared to patients whose urinary sTREM-1 levels were below the limit of quantification. Conclusion: Our present study suggested that TREM-1 in clgA1 induced inflammatory kidney injury in IgAN.

(c) 2018 S. Karger AG, Basel

\section{Introduction}

Immunoglobulin A nephropathy (IgAN) represents a common type of primary glomerulonephritis with the characterization of mesangial IgA deposition $[1,2]$. Approximately $30-40 \%$ of patients with IgAN progress to end-stage renal disease in 10-20 years [3]. The pathogenesis of IgAN is still unclear today. However, it is well accepted that circulating IgA1-containing immune com-

\footnotetext{
Li Zhu

Renal Division, Department of Medicine, Peking University First Hospital Peking University Institute of Nephrology

No 8, Xishiku Street, Xicheng District, Beijing 100034 (China)

E-Mail funnyzhuli@bjmu.edu.cn
} 
plexes (cIgA1) play an important role in the initiation of glomerular injury in IgAN. They could induce the local activation of the complement system and mesangial cells and upregulate the secretion of extracellular matrix and various inflammatory factors, such as MCP-1, IL-6, IL-8, and TNF- $\alpha[4,5]$. The inflammatory factors derived from mesangial cells could further induce damage to other intrinsic renal cells, such as podocytes and tubular epithelial cells [6-8]. Accordingly, higher urinary MCP-1 and IL-6 levels were reported to be associated with poor prognosis in patients with IgAN $[9,10]$, which implied the renal local inflammatory status in patients with IgAN. Therefore, inflammation was regarded as a major contributor to kidney injury in IgAN.

Our previous study reported the upregulation of multiple biological factors derived from cultured mesangial cells after challenge by cIgA1 isolated from IgAN patients, which included MCP-1, IL-6, CXCL1, and soluble triggering receptor expressed on myeloid cells-1 (sTREM-1) [11]. Among these factors, sTREM-1 came from the extracellular domains' shedding of TREM-1 [12]. TREM-1 is an activating receptor of immunoglobulin superfamily members. It is usually expressed on neutrophils and monocytes [13]. Although the exact ligand for TREM- 1 is unknown today, TREM- 1 activation could induce $\mathrm{Ca}^{2+}$ mobilization, activate the MARK/ERK and $\mathrm{NF}-\kappa \mathrm{B}$ signaling pathway, and upregulate the secretion of IL-8, MCP-1, and TNF- $\alpha$ [14]. Thus, TREM- 1 is regarded as an inflammatory amplifier that contributes to the initiation and amplification of the inflammatory response.

In inflammatory diseases induced by infection, such as sepsis and chronic obstructive pulmonary disease, the expression of TREM-1 is upregulated on neutrophils and monocytes, which amplifies inflammation and exacerbates tissue damage [15-17]. Recent studies have found that the expression of TREM-1 was not restricted to myeloid cells, as hepatic endothelial cells in endotoxemia and gastric epithelial cells stimulated by Helicobacter pylori also had TREM-1 expression [18]. Furthermore, in autoimmune inflammatory disease, such as rheumatoid arthritis and inflammatory bowel disease, TREM-1 also participated in local immune inflammatory injury and was correlated with disease activity [19-22]. Moreover, elevated sTREM-1 levels were reported in the plasma of patients with end-stage renal disease and the urine of patients with acute kidney injury, which suggested that TREM-1 might also play some role in kidney disease [23, 24].

IgAN is a type of autoimmune glomerulonephritis in which glomerular injury caused by inflammation plays an important role in disease progression. Based on the im- portant role of TREM-1 in the regulation of inflammation, in the present study, we explored whether TREM-1 was involved in kidney damage in IgAN and further investigated the role of urinary sTREM-1 detection in monitoring IgAN severity.

\section{Materials and Methods}

\section{Study Population}

In total, 35 patients with IgAN (18 males and 17 females, age: $36.7 \pm 13.3$ years) diagnosed in April 2013 in Peking University First Hospital were recruited in our present study. The diagnosis of IgAN was based on the presence of dominant IgA deposition in the mesangial area by immunofluorescence and electron-dense material deposition in the mesangial area by electronic microscopy. Patients with Henoch-Schönlein purpura, liver cirrhosis, and other secondary etiologies of IgAN were excluded by detailed clinical and laboratory examinations. Accordingly, 17 age- and gender-matched healthy volunteers ( 7 males and 10 females, age: 33.3 \pm 7.8 years) were recruited as controls.

The study protocol was reviewed and approved by the Ethics Committee of Peking University First Hospital, and written informed consent was obtained from all participants.

\section{Information on Clinical and Pathological Manifestations}

For enrolled patients, clinical manifestations, including age, gender, blood pressure, serum creatinine, 24 -h urine protein, and the proportion of crescentic glomeruli, were collected from medical records. The glomerular filtration rate was estimated according to the Chronic Kidney Disease Epidemiology Collaboration equation [25]. For the evaluation of pathological lesions, the Oxford classification [26] was scored by a pathologist who was blinded to patients' clinical baseline and outcome information.

\section{Isolation of $\operatorname{cIgA} 1$}

For recruited individuals, $10 \mathrm{~mL}$ of peripheral venous blood (EDTA anticoagulated) was collected on the morning of the renal biopsy (for IgAN patients) or recruitment (for healthy controls [HC]). Then, plasma was collected by centrifugation, divided into aliquots, and stored at $-80^{\circ} \mathrm{C}$ until the isolation of IgA 1 complexes. For our 35 recruited patients with IgAN and the 17 healthy volunteers, the IgA1 complexes were isolated separately from the plasma samples of each individual without pool plasma samples by jacalin affinity chromatography and then Sephacryl S-300 gel filtration chromatography, as previously reported [11].

\section{Cell Culture Experiments}

Primary human glomerular mesangial cells were purchased from ScienCell ${ }^{\mathrm{TM}}$ Corporation (ScienCell ${ }^{\mathrm{TM}}$, Carlsbad, CA, USA). Cells were cultured according to the manufacturer's specifications in complete culture medium (mesangial cell medium supplemented with 5\% FBS, mesangial cell growth supplement, penicillin G $[100 \mathrm{U} / \mathrm{mL}]$, and streptomycin $[100 \mathrm{U} / \mathrm{mL}])$ at $37^{\circ} \mathrm{C}$ in a humidified $5 \% \mathrm{CO}_{2}$ incubator. After overnight serum starvation, human mesangial cells were incubated with $100 \mu \mathrm{g} / \mathrm{mL}$ of cIgA1 from IgAN patients or $\mathrm{HC}$ for $48 \mathrm{~h}$. After centrifugation, supernatants of the cultured mesangial cells were collected and stored at $-80^{\circ} \mathrm{C}$ until detection. 
Fig. 1. Circulating IgA1-containing immune complexes (cIgA1) activated human mesangial cells. Compared to those from healthy controls, cIgA 1 from patients with IgA nephropathy significantly upregulated human mesangial cell proliferation (a; 1.15 \pm 0.08 vs. $1.08 \pm 0.06, p=0.002$ ) as well as secretion of MCP-1 (b; $1,470.4 \pm 264.6$ vs. $1,028.5 \pm 166.0 \mathrm{pg} / \mathrm{mL}, p<0.001)$ and IL-6 (c; $53.7[48.7,65.7]$ vs. $47.0[41.8,52.1] \mathrm{pg} /$ $\mathrm{mL}, p=0.008)$. Moreover, levels of secreted MCP-1 and IL-6 from mesangial cells showed a positive correlation (d; correlation coefficient $=0.589, p<0.001$ ).

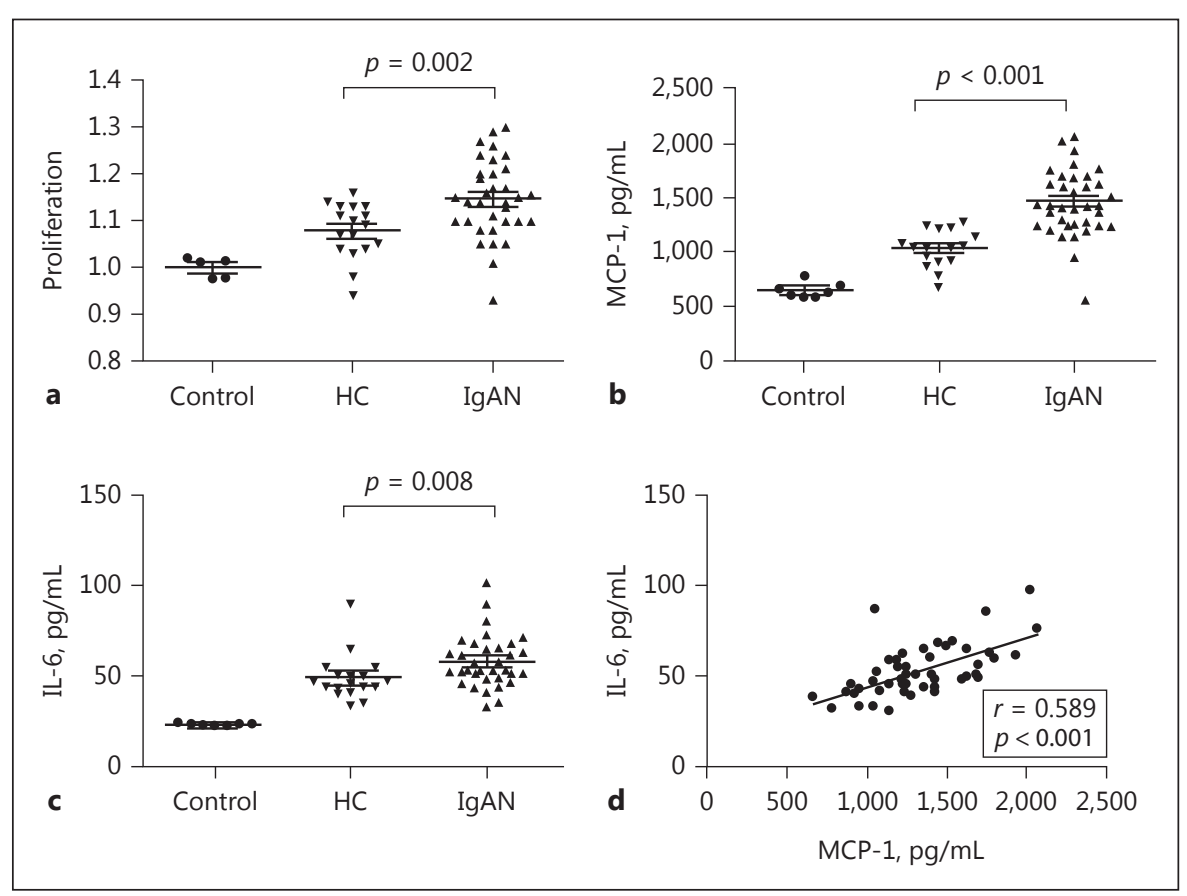

\section{Mesangial Cell Proliferation Assay}

Mesangial cells were seeded in 96-well plates before the experiments. After overnight serum starvation, mesangial cells were treated with $100 \mu \mathrm{g} / \mathrm{mL}$ of cIgA1 from IgAN patients or $\mathrm{HC}$ for $3 \mathrm{~h}$. Cell proliferation was evaluated by the MTS method using the CellTiter $96^{\circledR}$ AQueous One Solution Cell Proliferation Assay (Promega, Madison, WI, USA) according to the manufacturer's instructions.

Detection of Cytokine Levels by ELISA

The IL-6, CXCL1, MCP-1, and sTREM-1 levels in cell culture supernatants and urine samples were detected by standard sandwich ELISA assays using the DuoSet human ELISA kits (R\&D Systems, Minneapolis, MN, USA) according to the manufacturer's specifications. Urinary MCP-1, IL-6, CXCL1, and sTREM-1 levels were calibrated against urine creatinine before the comparison between IgAN patients and HC.

\section{RNA Isolation, Reverse Transcription and Real-Time}

Quantitative PCR

After cultured mesangial cells were extracted by the TRIZOL ${ }^{\circledR}$ Reagent (Invitrogen, USA), total RNA was reverse-transcribed to cDNA by a Reverse Transcription System (Promega). The cDNA was stored at $-20^{\circ} \mathrm{C}$ before further amplification. Gene expression levels of TREM-1, MMP2, MMP9, and GAPDH were examined by real-time PCR using Taqman gene expression assays (Applied Biosystems, USA; TREM-1: Hs00218624_m1, MMP2: Hs01548727_ m1, MMP9: Hs00957562_m1, GAPDH: Hs02758991_g1) on an Applied Biosystem VitA ${ }^{\mathrm{TM}} 7$ Real-Time PCR System. The relative expression of $\mathrm{mRNA}$ was calculated using the following formula: $2^{-\Delta \Delta \mathrm{Ct}}$, in which $\Delta \mathrm{Ct}=\mathrm{Ct}_{\text {objective gene }}-\Delta \mathrm{Ct}_{\mathrm{GAPDH}}$, while $\Delta \Delta \mathrm{Ct}=$ $\Delta \mathrm{Ct}_{\text {experimental group }}-\Delta \mathrm{Ct}_{\text {control group }}$.

\section{Statistical Analyses}

Statistical analyses were performed using SPSS software (version 18.0; SPSS, Chicago, IL, USA). For data description, normally distributed quantitative variables were expressed as the mean \pm standard deviation, while for non-normally distributed variables, the median and interquartile range were used. Categorical data were summarized as absolute frequencies and percentages. For comparison between groups, the independent-samples $t$ test was used if the data were normally distributed, and if not, Mann-Whitney tests were performed. Spearman's correlation was applied to analyze correlations. A two-tailed $p$ value $<0.05$ was considered significant.

\section{Results}

\section{cIgA1 Derived from IgAN Patients Induced the}

\section{Activation of Human Mesangial Cells}

In IgAN, deposits of IgA are mainly observed in the mesangial area; thus, we isolated cIgA1 from 35 IgAN patients and $17 \mathrm{HC}$ to treat cultured human mesangial cells. The cIgA1 derived from both IgAN patients (IgANcIgA1) and HC (HC-cIgA1) induced the proliferation of mesangial cells and upregulated the excretion of the inflammatory cytokines MCP- 1 and IL- 6 compared to nonIgA1 controls. Furthermore, the degree of mesangial cell proliferation and the levels of IL- 6 and MCP-1 were significantly higher in the IgAN-cIgA1 group than in the 


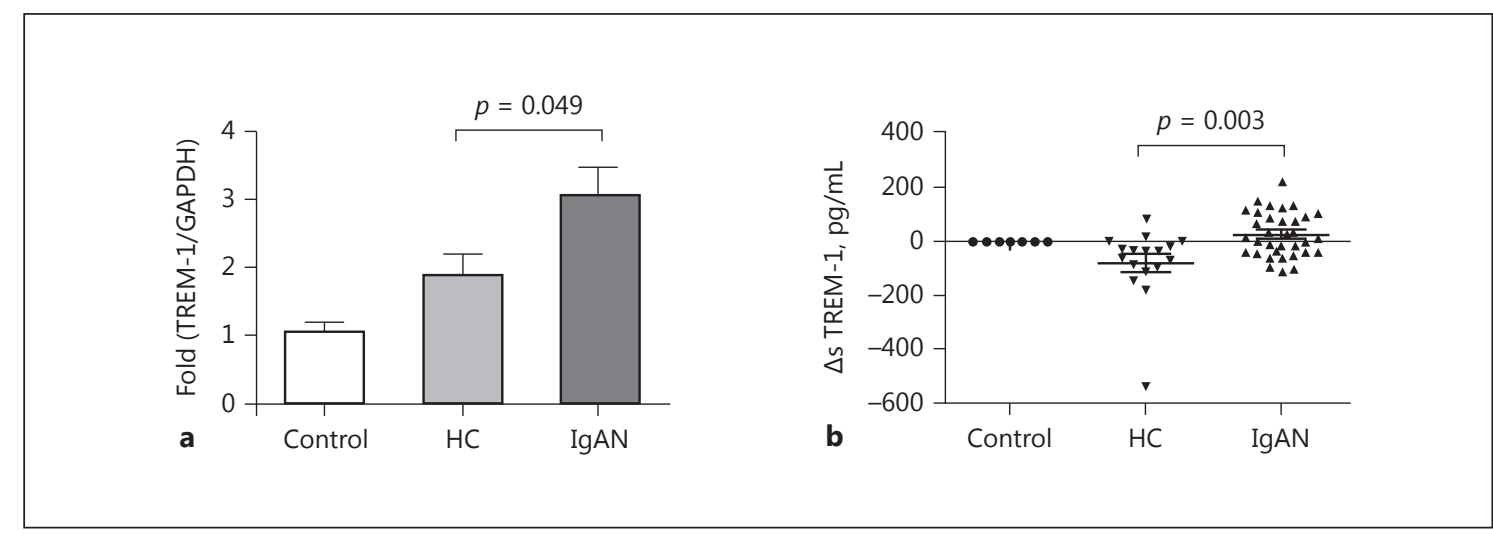

Fig. 2. Circulating IgA1-containing immune complexes (cIgA1) from IgA nephropathy (IgAN) patients induced upregulated TREM-1 expression in mesangial cells. a The mRNA expression of TREM-1 in human mesangial cells was significantly upregulated by cIgA1 derived from IgAN patients $(2.39$ [1.35, 4.24] vs. 1.50

HC-cIgA1 group (proliferation: $1.15 \pm 0.08$ vs. $1.08 \pm$ $0.06, p=0.002$; MCP $-1: 1,470.4 \pm 264.6$ vs. $1,028.5 \pm 166.0$ $\mathrm{pg} / \mathrm{mL}, p<0.001$; IL-6: 53.7 [48.7, 65.7] vs. 47.0 [41.8, $52.1] \mathrm{pg} / \mathrm{mL}, p=0.008$; Fig. 1 ), which indicated that cIgA1 derived from IgAN patients could induce the activation of cultured human mesangial cells, similar to the mesangial hypercellularity lesions observed in renal biopsy samples of patients with IgAN. Moreover, levels of the 2 inflammatory cytokines, MCP-1 and IL-6, correlated well with each other (correlation coefficient $=0.589, p<0.001$; Fig. 1).

\section{cIgA1 Derived from IgAN Patients Upregulated}

TREM-1 Expression in Human Mesangial Cells

To evaluate the involvement of TREM- 1 in the process of mesangial cell activation in IgAN, we detected the mRNA expression of TREM-1 in mesangial cells and sTREM-1 levels in culture supernatant under CIgA1 challenge. Our results indicated that the expression of TREM1 in human mesangial cells was significantly upregulated by cIgA1 derived from IgAN patients, as indicated by higher mRNA levels of TREM-1 in human mesangial cells (IgAN-cIgA1 vs. HC-cIgA1: 2.39 [1.35, 4.24] vs. 1.50 $[1.18,1.85], p=0.049$; Fig. 2a), as well as elevated $\triangle$ sTREM-1 levels in the supernatant of mesangial cells (IgAN-cIgA1 vs. HC-cIgA1: $18.7[-36.1,97.1]$ vs. -34.5 $[-95.8,9.1] \mathrm{pg} / \mathrm{mL}, p=0.003$; Fig. 2b).

It was proposed that sTREM-1 originated from extracellular domains' shedding of TREM-1 by matrix metalloproteinases [12]. Here, we detected the expression of 2
$[1.18,1.85], p=0.049)$. b Additionally, the levels of $\Delta$ sTREM-1 in the supernatant of mesangial cells were also significantly higher after treatment with cIgA1 derived from IgAN patients, compared to those from healthy controls $(18.7[-36.1,97.1]$ vs. $-34.5[-95.8$, 9.1] $\mathrm{pg} / \mathrm{mL}, p=0.003)$. kinds of matrix metalloproteinases, MMP9 and MMP2, in human mesangial cells. Our results showed that compared to HC-cIgA1, IgAN-cIgA1 could significantly upregulate the mRNA expression of MMP9 in mesangial cells (IgAN-cIgA1 vs. HC-cIgA1: $1.01[0.76,1.99]$ vs. 0.57 $[0.41,0.97], p=0.003$; Fig. 3a). Moreover, the expression of MMP9 and TREM-1 showed a positive correlation in mesangial cells after treatment with $\operatorname{cIgA1}(r=0.551, p<$ 0.001; Fig. 3b). Regarding MMP2, no significant differences were observed between the IgAN-cIgA1 group and the HC-cIgA1 group (IgAN-cIgA1 vs. HC-cIgA1: 0.97 $[0.84,1.19]$ vs. $0.94[0.75,1.02], p=0.242$; Fig. $3 c)$.

\section{The Expression of TREM-1 Is Correlated with the} Activation of Human Mesangial Cells

After the identification of upregulated TREM-1 expression in mesangial cells under cIgA1 treatment, we next investigated the involvement of TREM-1 in mesangial cell activation. Positive correlations were observed between mesangial cell proliferation and the mRNA expression of TREM-1 (correlation coefficient $=0.315, p=$ 0.023; Fig. 4a) as well as $\triangle$ sTREM-1 levels (correlation coefficient $=0.347, p=0.012$; Fig. $4 \mathrm{~b}$ ) in the supernatant of cultured mesangial cells. In addition, MCP-1 levels, but not IL-6 levels, showed a positive correlation with $\triangle$ sTREM-1 levels (MCP-1: correlation coefficient $=0.407$, $p=0.003$; IL-6: correlation coefficient $=-0.092, p=0.517$; Fig. 5). Our results suggested that upregulated TREM-1 might contribute to human mesangial cell activation under the challenge of pathogenic cIgA1. 
Fig. 3. Circulating IgA1-containing immune complexes (cIgA1) from IgA nephropathy (IgAN) patients induced upregulated MMP9 expression in mesangial cells. a The mRNA expression of MMP9 in human mesangial cells was significantly upregulated by cIgA1 derived from IgAN patients $(1.01[0.76,1.99]$ vs. 0.57 [0.41, 0.97], $p=0.003)$. b Moreover, the expression of MMP9 and TREM-1 showed positive correlation in mesangial cells after cIgA1 treatment $(r=0.551, p<0.001)$. However, the expression of MMP2 was comparable between the IgAN-cIgA1 group and the healthy control (HC)-cIgA1 group (c; $0.97[0.84,1.19]$ vs. 0.94 [0.75, 1.02], $p=0.242)$.

Fig. 4. The expression of TREM-1 correlated with activation of mesangial cells. Mesangial cell proliferation showed a positive correlation with both mRNA expression of TREM-1 (a; correlation coefficient $=0.315, p=0.023)$ and $\Delta$ sTREM-1 levels in the supernatant of cultured mesangial cells $(\mathbf{b}$; correlation coefficient $=$ $0.347, p=0.012$ ).

Fig. 5. Levels of $\triangle$ sTREM-1 correlated with MCP-1 levels derived from mesangial cells after circulating IgA1-containing immune complexes treatment. The MCP-1 levels (a; correlation coefficient $=0.407, p=0.003$ ), but not IL-6 levels (b; correlation coefficient $=-0.092, p=0.517)$, showed a positive correlation with $\triangle$ sTREM-1 levels.
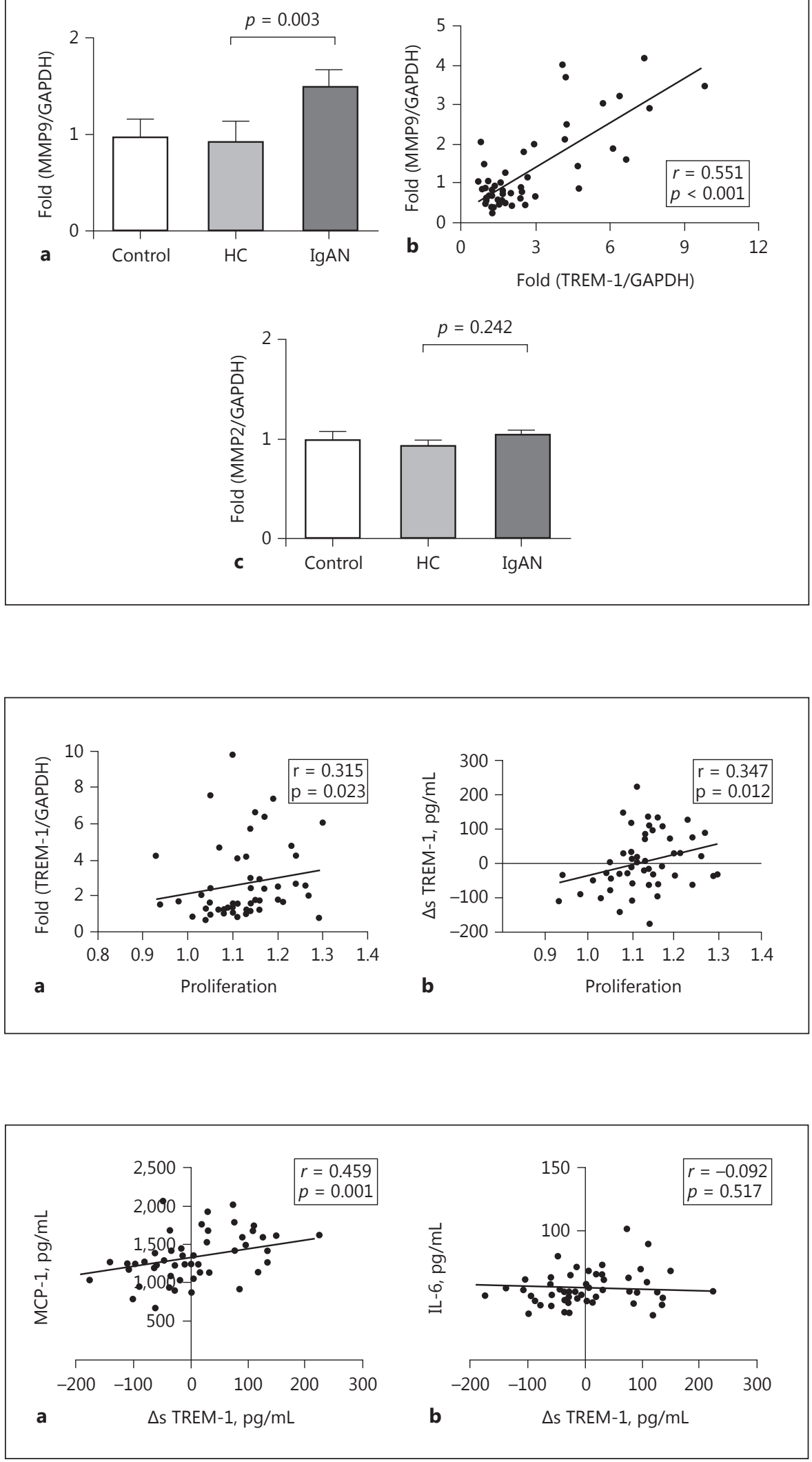
Table 1. Baseline clinical and laboratory data in patients with IgA nephropathy

\begin{tabular}{|c|c|c|c|c|}
\hline \multirow[t]{2}{*}{ Parameters } & \multirow{2}{*}{$\begin{array}{l}\text { All patients } \\
(n=35)\end{array}$} & \multicolumn{2}{|l|}{ Urinary TREM-1 } & \multirow{2}{*}{$\begin{array}{l}p \\
\text { value }\end{array}$} \\
\hline & & $\begin{array}{l}\text { below LOD group } \\
(n=23)\end{array}$ & $\begin{array}{l}\text { detectable group } \\
(n=12)\end{array}$ & \\
\hline \multicolumn{5}{|l|}{ Clinical indicators } \\
\hline Age, years & $36.7 \pm 13.3$ & $33.2 \pm 11.3$ & $43.3 \pm 14.7$ & 0.065 \\
\hline Gender (male/female) & $18 / 17$ & $11 / 12$ & $7 / 5$ & 0.815 \\
\hline 24-h urine protein, g/day & $1.72(0.64,2.71)$ & $1.07(0.82,2.37)$ & $2.08(0.42,4.19)$ & 0.469 \\
\hline$<0.3 \mathrm{~g} /$ day & $3 / 33(9.1)$ & $2(8.7)$ & $1(10.0)$ & \\
\hline $0.3-0.99 \mathrm{~g} / \mathrm{day}$ & $10 / 33(30.3)$ & $7(30.4)$ & $3(30.0)$ & \\
\hline $1.0-2.99 \mathrm{~g} /$ day & $14 / 33(42.4)$ & $12(52.2)$ & $2(20.0)$ & \\
\hline$\geq 3.0 \mathrm{~g} /$ day & $6 / 33(18.2)$ & $2(8.7)$ & $4(40.0)$ & \\
\hline $\mathrm{eGFR}, \mathrm{mL} / \mathrm{min} / 1.73 \mathrm{~m}^{2}$ & $76.3 \pm 39.1$ & $86.4 \pm 34.1$ & $57.0 \pm 42.1$ & 0.065 \\
\hline $\mathrm{MAP}, \mathrm{mm} \mathrm{Hg}$ & $94 \pm 13$ & $93 \pm 12$ & $102 \pm 12$ & 0.037 \\
\hline Serum IgA, g/L & $3.59 \pm 1.09$ & $3.28 \pm 0.97$ & $4.23 \pm 1.08$ & 0.028 \\
\hline \multicolumn{5}{|l|}{ Pathological indicators } \\
\hline \multicolumn{5}{|l|}{ Crescents, \% } \\
\hline Cellular crescents & $2.9(0.0,7.9)$ & $2.9(0.0,6.1)$ & $3.1(0.0,9.8)$ & 0.38 \\
\hline Cellular fibrous & $1.9(0.0,12.1)$ & $0.0(0.0,6.5)$ & $4.5(0.0,2.7)$ & 0.268 \\
\hline Fibrous & $0.0(0.0,0.0)$ & $0.0(0.0,0.0)$ & $0.0(0.0,3.5)$ & 0.354 \\
\hline \multicolumn{5}{|l|}{ Oxford classification $^{\mathrm{a}}$} \\
\hline M1 & $27(79.4)$ & $19(82.6)$ & $8(72.7)$ & 0.656 \\
\hline E1 & $9(26.5)$ & $5(21.7)$ & $4(36.4)$ & 0.425 \\
\hline S1 & $22(64.7)$ & $17(73.9)$ & $5(45.5)$ & 0.138 \\
\hline $\mathrm{T} 1 / \mathrm{T} 2$ & $14(41.2) / 10(29.4)$ & $9(39.1) / 6(26.1)$ & $5(45.5) / 4(36.4)$ & 0.596 \\
\hline
\end{tabular}

Values are presented as mean \pm standard deviation, median (interquartile range), or $n$ (\%), unless otherwise indicated. LOD, limit of detection; eGFR, estimated glomerular filtration rate; MAP, mean arterial pressure. Oxford classification: mesangial hypercellularity score $(\mathrm{M} 1>0.5)$, presence of endocapillary proliferation (E1: present), segmental glomerulosclerosis/adhesion (S1: present), and severity of tubular atrophy/interstitial fibrosis (T1: $26-50 \%$, T2: $>50 \%)$.

a The Oxford classification was developed by the Working Group of the International IgA Nephropathy Network and the Renal Pathology Society.

Elevated Urinary sTREM-1 Levels in Patients with IgAN Since urine samples contain many inflammatory chemokines and cytokines derived from the kidney, we next detected urinary sTREM-1 levels in our recruited individuals. The sTREM-1 levels in the urine samples were very low. Urinary sTREM-1 levels in all our recruited $\mathrm{HC}$ were below the limit of detection (LOD), while $34.3 \%(12 / 35)$ of IgAN patients showed detectable urinary sTREM- 1 levels, and the other $65.7 \%$ (23/35) were below the LOD. Therefore, the group of IgAN patients showed higher urinary sTREM-1 levels compared to $\mathrm{HC}(0[0,57.90]$ vs. $0[0,0] \mathrm{pg} / \mathrm{mg}, p=0.004)$.

Then, we further explored the association of urinary sTREM-1 levels with clinical and pathological manifestations, as well as levels of urinary inflammatory factors in patients with IgAN. Since a considerable number of patients with IgAN had low urinary sTREM-1 levels that were below the LOD of ELISA, we classified our recruited patients with IgAN into 2 subgroups according to their urinary sTREM-1 levels that were below those of the LOD group and the detectable group. We found that $\Delta$ sTREM-1 levels in the supernatant of mesangial cells challenged by cIgA 1 from IgAN patients in the detectable group showed a higher trend compared to those from the below LOD group. Moreover, patients in the detectable group presented with significantly higher plasma IgA levels and lower estimated glomerular filtration rate (eGFR) levels compared to the other patients in the below LOD group (IgA: $4.23 \pm 1.08$ vs. $3.28 \pm 0.97 \mathrm{~g} / \mathrm{L}, p=0.028$; eGFR: 57.00 \pm 42.15 vs. $86.40 \pm 34.09 \mathrm{~mL} / \mathrm{min} / 1.73 \mathrm{~m}^{2}, p=0.032 ; \mathrm{Ta}-$ ble 1). However, for patients with detectable urinary sTREM-1, no significant correlation was found between urine TREM-1 levels and plasma IgA levels or eGFR levels. Our results implied that elevated urinary sTREM-1 levels were correlated with severe phenotypes in IgAN. 


\section{Discussion}

TREM- 1 is upregulated and participates in tissue damage due to its amplification of the inflammatory response in various diseases, including sepsis, inflammatory bowel disease, and rheumatoid arthritis $[15,19,20]$. In our study, we found that TREM-1 was upregulated in patients with IgAN, and the elevated TREM-1 levels were associated with the activation of mesangial cells, which implicated the involvement of TREM-1 in the pathogenesis of IgAN.

Previous studies revealed that the cross-linking of TREM-1 induced the secretion of inflammatory factors, including MCP-1, IL- 8 , and TNF- $\alpha$, in neutrophils and monocytes, demonstrating the importance of TREM-1's action as an upstream regulatory factor to amplify inflammatory responses [13]. In the present study, using cultured mesangial cells challenged by cIgA1 as the cell model of IgAN, we observed elevated levels of MCP-1 and IL-6 in the mesangial cell supernatant, which was in accordance with previous reports $[4,27]$. Furthermore, we also found the upregulated expression of TREM-1 in mesangial cells after challenge by cIgA1, and the TREM-1 levels were positively correlated with levels of MCP-1 in the mesangial supernatant, which suggested that TREM-1 might play a role in the regulation of mesangial cell-derived inflammatory factor secretion in IgAN.

Since the blockade of TREM-1 was reported to be an effective treatment method in animal models of inflammation, such as mice challenged with LPS [15] and the DSS model of colitis [22], the modulation of TREM-1 engagement might represent a novel therapeutic approach for the treatment of diseases through the suppression of inflammation. Therefore, based on our results of the induction of TREM-1 expression in mesangial cells after challenge of the pathogenic IgA1-containing immune complexes, we speculated that TREM-1 may be a potential therapeutic target for the treatment of IgAN.

The structure of sTREM-1 is similar to that of the extracellular domain of TREM-1, and the inhibition of MMP could upregulate the stability of membranous TREM-1 and reduce the release of sTREM-1, implying that MMP might break down the extracellular domain of TREM-1 into sTREM-1 [12]. It was reported that in experimental mice with IgAN, the expression of MMP9 in renal tissues was higher compared to that in HC. In our study, we also observed elevated MMP9 expression in the IgAN group, and the MMP9 expression levels were associated with TREM-1 expression. Our results suggested that in IgAN, MMP9 might serve as the "tool" to break down the extracellular domain of TREM-1 into sTREM-1.

In some patients with IgAN, we also found that their urinary sTREM-1 levels were higher than those of the $\mathrm{HC}$, represented by about one-third of patients with IgAN with detectable urinary sTREM-1 levels, while no HC showed detectable urinary sTREM-1 levels. Furthermore, our IgAN patients with detectable urinary sTREM-1 levels showed severe clinical phenotypes as well as higher urinary inflammatory factor MCP-1 levels, which implied that urinary sTREM-1 levels could reflect disease severity and the local renal inflammation status in IgAN. Thus, urinary sTREM-1 may be a potential noninvasive biomarker for IgAN, and their clinical value requires further verification in independent IgAN cohorts.

Our study also has a few limitations. Firstly, the involvement of TREM-1 in IgAN should be confirmed by blocking experiments. However, because of the limited quantity of IgA1-containing immune complexes isolated from circulation, we did not have enough IgA1-containing immune complexes for further experiments. Secondly, it was reported that urinary sTREM-1 levels are higher in patients with other kidney diseases, such as anti-GBM disease and acute kidney injury [24, 28]; thus, it could not be a specific biomarker for IgAN. More studies are needed to evaluate its potential clinical implications in these kidney diseases.

In conclusion, in the present study, our results indicated the contribution of TREM-1 in inflammatory kidney injury in IgAN induced by cIgA1 and further identified the potential clinical value of urinary sTREM- 1 as a noninvasive biomarker to reflect IgAN severity. These findings suggested that TREM-1 might be a potential therapeutic target for the treatment of IgAN.

\section{Acknowledgement}

This work was supported by grants from National Science Foundation of China (Grant No. 81470945;81670638); the Training Program of the Major Research Plan of the National Natural Science Foundation of China (91642120); Capital of Clinical Characteristics and the Applied Research Fund (Z141107002514037, Z161100000516005);, Beijing New-Star Plan of Science and Technology (Z161100004916167); and the National Key Research and Development Program of China (2016YFC0904102).

\section{Conflict of Interest Statement}

The authors have no conflicts of interest to disclose. 


\section{References}

1 D'Amico G: The commonest glomerulonephritis in the world: IgA nephropathy. Q J Med 1987;64:709-727.

2 Donadio JV, Grande JP: IgA nephropathy. N Engl J Med 2002;347:738-748.

3 Schena FP: A retrospective analysis of the natural history of primary $\operatorname{IgA}$ nephropathy worldwide. Am J Med 1990;89:209-215.

4 Novak J, Raskova KL, Suzuki H, Tomana M, Matousovic K, Brown R, Hall S, Sanders JT, Eison TM, Moldoveanu Z, Novak L, Novak Z, Mayne R, Julian BA, Mestecky J, Wyatt RJ: IgA1 immune complexes from pediatric patients with IgA nephropathy activate cultured human mesangial cells. Nephrol Dial Transplant 2011;26:3451-3457.

5 Novak J, Tomana M, Matousovic K, Brown R, Hall S, Novak L, Julian BA, Wyatt RJ, Mestecky J: IgA1-containing immune complexes in IgA nephropathy differentially affect proliferation of mesangial cells. Kidney Int 2005; 67:504-513.

6 Lai KN, Leung JC, Chan LY, Saleem MA, Mathieson PW, Tam KY, Xiao J, Lai FM, Tang SC: Podocyte injury induced by mesangialderived cytokines in IgA nephropathy. Nephrol Dial Transplant 2009;24:62-72.

7 Lai KN, Leung JC, Chan LY, Saleem MA, Mathieson PW, Lai FM, Tang SC: Activation of podocytes by mesangial-derived TNF-alpha: glomerulo-podocytic communication in IgA nephropathy. Am J Physiol Renal Physiol 2008;294:F945-F955.

8 Lai KN, Tang SC, Leung JC: Recent advances in IgA nephropathy - the glomerulopodocytic-tubular communication. Adv Otorhinolaryngol 2011;72:40-44.

9 Stangou M, Alexopoulos E, Papagianni A, Pantzaki A, Bantis C, Dovas S, Economidou D, Leontsini M, Memmos D: Urinary levels of epidermal growth factor, interleukin- 6 and monocyte chemoattractant protein-1 may act as predictor markers of renal function outcome in immunoglobulin A nephropathy. Nephrology (Carlton) 2009;14:613-620.

10 Ranieri E, Gesualdo L, Petrarulo F, Schena FP: Urinary IL-6/EGF ratio: a useful prognostic marker for the progression of renal damage in IgA nephropathy. Kidney Int 1996;50:19902001.

11 Zhu L, Zhang Q, Shi S, Liu L, Lv J, Zhang H: Synergistic effect of mesangial cell-induced CXCL1 and TGF-beta1 in promoting podocyte loss in IgA nephropathy. PLoS One 2013; 8:e73425.
12 Gómez-Piña V, Soares-Schanoski A, Rodríguez-Rojas A, Del Fresno C, García F, VallejoCremades MT, Fernández-Ruiz I, Arnalich F, Fuentes-Prior P, López-Collazo E: Metalloproteinases shed TREM-1 ectodomain from lipopolysaccharide-stimulated human monocytes. J Immunol 2007;179:4065-4073.

13 Bouchon A, Dietrich J, Colonna M: Cutting edge: inflammatory responses can be triggered by TREM-1, a novel receptor expressed on neutrophils and monocytes. J Immunol 2000; 164:4991-4995.

14 Tessarz AS, Cerwenka A: The TREM-1/ DAP12 pathway. Immunol Lett 2008;116: 111-116.

15 Bouchon A, Facchetti F, Weigand MA, Colonna M: TREM-1 amplifies inflammation and is a crucial mediator of septic shock. Nature 2001;410:1103-1107.

16 Knapp S, Gibot S, de Vos A, Versteeg HH, Colonna M, van der Poll T: Cutting edge: expression patterns of surface and soluble triggering receptor expressed on myeloid cells- 1 in human endotoxemia. J Immunol 2004;173: 7131-7134.

17 Radsak MP, Taube C, Haselmayer P, Tenzer S, Salih HR, Wiewrodt R, Buhl R, Schild H. Soluble triggering receptor expressed on myeloid cells 1 is released in patients with stable chronic obstructive pulmonary disease. Clin Dev Immunol 2007;2007:52040.

18 Schmausser B, Endrich S, Beier D, Moran AP, Burek CJ, Rosenwald A, Rieckmann P, Müller-Hermelink HK, Eck M: Triggering receptor expressed on myeloid cells-1 (TREM-1) expression on gastric epithelium: implication for a role of TREM-1 in Helicobacter pylori infection. Clin Exp Immunol 2008;152:8894.

19 Park JJ, Cheon JH, Kim BY, Kim DH, Kim ES, Kim TI, Lee KR, Kim WH: Correlation of serum-soluble triggering receptor expressed on myeloid cells- 1 with clinical disease activity in inflammatory bowel disease. Dig Dis Sci 2009; 54:1525-1531.

20 Kim TH, Choi SJ, Lee YH, Song GG, Ji JD: Soluble triggering receptor expressed on myeloid cells- 1 as a new therapeutic molecule in rheumatoid arthritis. Med Hypotheses 2012; 78:270-272.
21 Murakami Y, Akahoshi T, Hayashi I, Endo H, Kawai S, Inoue M, Kondo H, Kitasato H: Induction of triggering receptor expressed on myeloid cells 1 in murine resident peritoneal macrophages by monosodium urate monohydrate crystals. Arthritis Rheum 2006;54: 455-462.

22 Schenk M, Bouchon A, Seibold F, Mueller C: TREM-1-expressing intestinal macrophages crucially amplify chronic inflammation in experimental colitis and inflammatory bowel diseases. J Clin Invest 2007;117:3097-3106.

23 Essa ES, Elzorkany KM: sTREM-1 in patients with chronic kidney disease on hemodialysis. APMIS 2015;123:969-974.

24 Su L, Xie L, Liu D: Urine sTREM-1 may be a valuable biomarker in diagnosis and prognosis of sepsis-associated acute kidney injury. Crit Care 2015;19:281.

25 Levey AS, Stevens LA, Schmid CH, Zhang YL, Castro AF 3rd, Feldman HI, Kusek JW, Eggers P, Van Lente F, Greene T, Coresh J; CKDEPI (Chronic Kidney Disease Epidemiology Collaboration): A new equation to estimate glomerular filtration rate. Ann Intern Med 2009;150:604-612.

26 Working Group of the International IgA Nephropathy Network and the Renal Pathology Society, Roberts IS, Cook HT, Troyanov S, Alpers CE, Amore A, Barratt J, Berthoux F, Bonsib S, Bruijn JA, Cattran DC, Coppo R, D'Agati V, D'Amico G, Emancipator S, Emma F, Feehally J, Ferrario F, Fervenza FC, Florquin S, Fogo A, Geddes CC, Groene HJ, Haas M, Herzenberg AM, Hill PA, Hogg RJ, Hsu SI, Jennette JC, Joh K, Julian BA, Kawamura T, Lai FM, Li LS, Li PK, Liu ZH, Mackinnon B, Mezzano S, Schena FP, Tomino Y, Walker PD, Wang H, Weening JJ, Yoshikawa $\mathrm{N}$, Zhang $\mathrm{H}$ : The Oxford classification of IgA nephropathy: pathology definitions, correlations, and reproducibility. Kidney Int 2009;76:546-556.

27 Tsuge T, Suzuki Y, Shimokawa T, Horikoshi S, Okumura K, Ra C, Tomino Y: Monocyte chemoattractant protein (MCP)-1 production via functionally reconstituted Fcalpha receptor (CD89) on glomerular mesangial cells. Inflamm Res 2003;52:428-432.

28 Du Y, Wu T, Zhou XJ, Davis LS, Mohan C: Blockade of CD354 (TREM-1) ameliorates anti-GBM-induced nephritis. Inflammation. 2016;39:1169-1176. 